\title{
Disseminated Nocardiosis Masquerading as an Obstructive Endobronchial Mass-A Case Report
}

\author{
Casey Morris, Luke Sorrell, Eitan Podgaetz, Adan Mora Jr., and Jeffrey Kopita \\ Department of Pulmonary and Critical Care Medicine, Baylor University Medical Center, Dallas, TX, USA
}

$\mathrm{P}$ resented is a rare case of disseminated nocardiosis in an immunocompetent, healthy, 70-year-old man. The patient initially presented to the clinic with unresolved pneumonia of 2 months duration, despite four courses of antibiotics. A non-contrasted chest computerized tomography (CT) demonstrated a right upper lobe endobronchial lesion with post-obstructive pneumonia and mediastinal lymphadenopathy. Flexible bronchoscopy revealed an obstructing, vascular mass involving the orifice of the right upper lobe anterior segment. Bronchoscopic cryotherapy was utilized to debulk the lesion. Four days later, he developed acute onset disequilibrium, confusion and headache. Imaging showed multiple bihemispheric ring-enhancing masses with diffusion restriction characteristic of intracranial abscesses and early signs of subfalcine herniation. He was empirically treated with broad-spectrum antibiotics and high-dose steroids. Tissue culture from bronchoscopy grew Nocardia the following day. Extensive work-up to elucidate occult immunosuppression was unrevealing. He was appropriately treated with triple therapy (sulfamethoxazole/trimethoprim, imipenem/cilastatin and linezolid), clinically improved and was discharged. The differential for a non-resolving pneumonia includes cancer and infection. Identification of concurrent brain lesions typically favors metastatic disease. This case illustrates the need to keep a broad differential on what may appear to be metastatic lung cancer.

\section{Keywords}

Nocardia, Nocardiosis, endobronchial mass, ring-enhancing mass, intracranial abscess

Disclosure: Casey Morris, Luke Sorrell, Eitan Podgaetz, Adan Mora Jr., and Jeffrey Kopita have no conflicts of interest to disclose in relation to this article.

Review Process: Double-blind peer review.

Compliance with Ethics: All procedures were followed in accordance with the responsible committee on human experimentation and with the Helsinki Declaration of 1975 and subsequent revisions. Written informed consent was not obtained from the patient case included in this report as no identifying information or images have been used.

Authorship: The named authors meet the International Committee of Medical Journal Editors (ICMJE) criteria for authorship of this manuscript, take responsibility for the integrity of the work as a whole, and have given final approval for the version to be published.

Received: August 2, 2019

Accepted: September 10, 2019

Citation: US Respiratory \& Pulmonary Diseases. 2019;4(1):20-3

Corresponding Author: Casey Morris, Baylor University Medical Center, 3500 Gaston Ave, Dallas, TX 75246 USA. E: Casey.Morris@BSWHealth.org

Twitter: @CaseyMorrisMD

Support: No funding was received in the publication of this article.

\section{Article Highlights}

- Nocardiosis has been coined "the great imitator" due to the vast heterogeneity in clinical presentation.

- Prevalence of disease is on the rise due to improved diagnostic techniques, as well as a growing population of immunocompromised patients, although up to one-third of patients diagnosed are immunocompetent.

- There are six forms of disease caused by Nocardia in humans: pulmonary nocardiosis, systemic (disseminated) nocardiosis (involving two or more body sites), central nervous system (CNS) nocardiosis, extrapulmonary nocardiosis, cutaneous and subcutaneous/ lymphocutaneous nocardiosis and actinomycetoma.

- Pulmonary nocardiosis may manifest as an endobronchial lesion which may imitate active malignancy.

- Having a high suspicion for CNS involvement is paramount, as up to $44 \%$ of patients will have CNS disease, which carries a mortality rate up to $64 \%$.

- For severe disease, including disseminated and CNS nocardiosis, intravenous sulfamethoxazole/trimethoprim, imipenem-cilastatin and amikacin is recommended.

Nocardiosis is a rare, opportunistic bacterial infection caused by Nocardia species that predominantly affects the respiratory tract of immunocompromised patients, although approximately one-third of infected patients are immunocompetent. ${ }^{1}$ The reported incidence of clinically significant infection has been increasing, likely attributable to improvement in diagnostic sensitivity, as well as increased prevalence of immunosuppressed patients and invasive surgical procedures. ${ }^{2}$ Due to the vast heterogeneity in symptomatology, diagnosis can be challenging. Because of the overlap in nonspecific clinical manifestations, occult malignancy is typically high in the differential.

Although rare, with only a few case reports, ${ }^{3}$ pulmonary nocardiosis may manifest as an endobronchial lesion that may be suggested on chest imaging and confirmed with fiberoptic bronchoscopy. This case represents the rare incidence of disseminated nocardiosis presenting with an endobronchial mass and the subsequent diagnosis of concomitant multiple intracranial abscesses 
Figure 1: Computed tomography chest

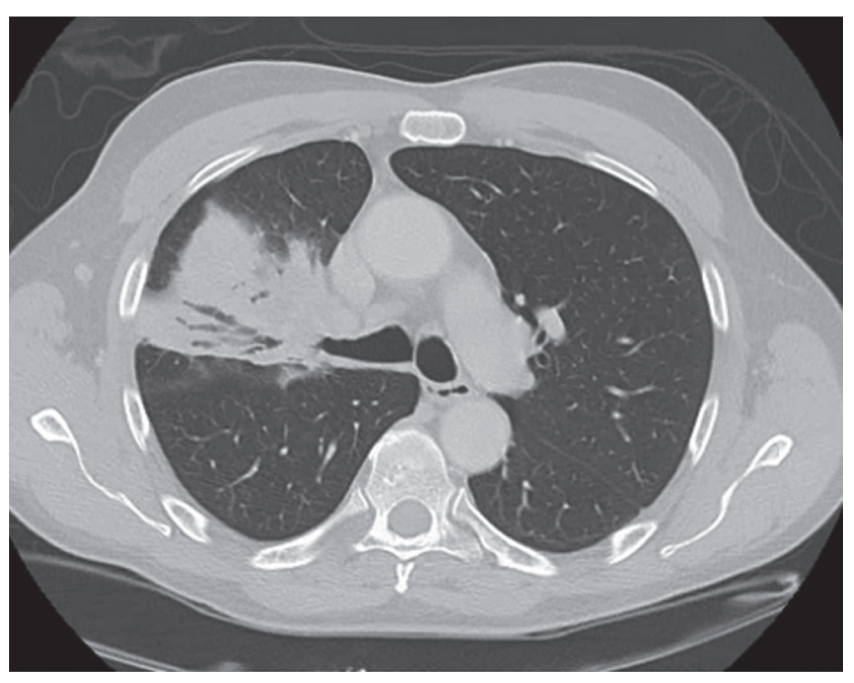

in an immunocompetent host. This illustrates the importance of having a high suspicion for nocardiosis, even in an immunocompetent host, as this disease carries a mortality rate of up to $64 \% .{ }^{4}$ It also stresses the value of obtaining appropriate and prompt central nervous system (CNS) imaging once the diagnosis is made to evaluate for disseminated disease.

\section{Case presentation}

A 70-year-old healthy male presented to clinic with unresolved pneumonia of 2 months duration, despite four separate courses of antibiotics. A non-contrasted computerized tomography (CT) demonstrated a right upper lobe endobronchial lesion with post-obstructive pneumonia and mediastinal lymphadenopathy (Figure 1). A flexible fiberoptic bronchoscopy was performed which revealed an obstructing, vascular mass originating from the orifice of the right upper lobe anterior segment with associated proximal occlusion of the entire right upper lobe (Figure 2).

Bronchoalveolar lavage, cytology and cultures (bacterial and fungal) were collected. Due to the vascular nature of the lesion, as well as severity of obstruction, bronchoscopic cryotherapy was utilized for both therapeutic debulking as well as obtaining tissue for diagnosis. Both histopathology as well as microbiology were tested. Initial cultures from bronchoalveolar lavage were unrevealing. Cytology was negative for malignancy, and pathology revealed only acute on chronic non-specific inflammation with no evidence of malignancy.

Four days later he developed acute onset disequilibrium, confusion and headache. He was advised to immediately present to the emergency department where a head CT revealed numerous supratentorial mass lesions with mass effect. A contrasted brain magnetic resonance imaging showed multiple bihemispheric ring-enhancing masses with diffusion restriction, characteristic of intracranial abscesses and early signs of subfalcine herniation (Figure 3).

He was admitted to the hospital and bolused $10 \mathrm{mg}$ of dexamethasone intravenously (IV) with subsequent dosing of $4 \mathrm{mg}$ IV every 6 hours. Initially, there was a high suspicion for active metastatic disease. Due to
Figure 2: Bronchoscopy right upper lobe

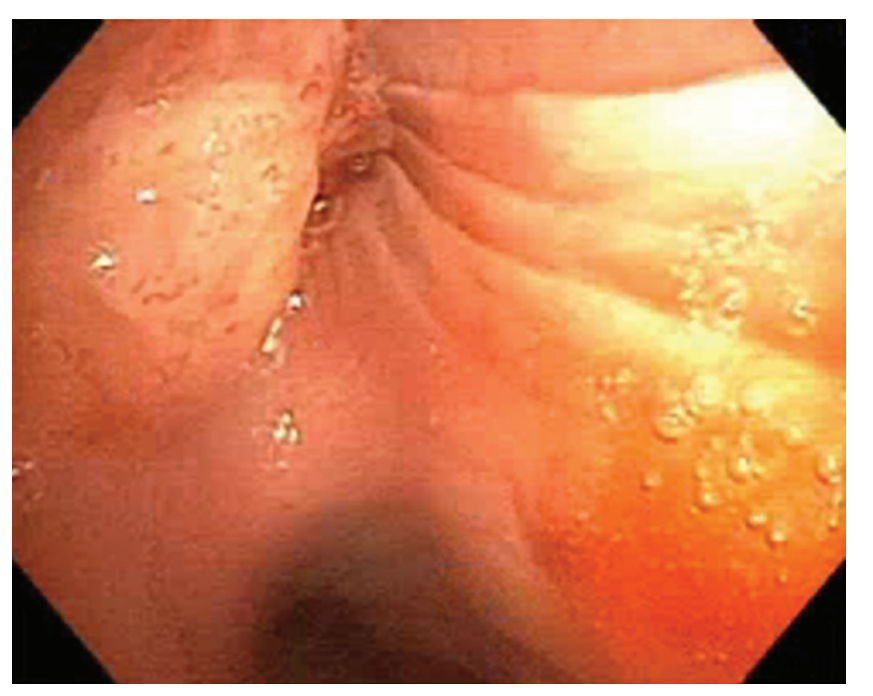

concerns for increased intracranial pressure, lumbar puncture was not performed, and neurosurgery was consulted to aide in obtaining tissue for diagnosis. On hospital day two, prior to brain biopsy, two separate tissue cultures from bronchoscopy grew filamentous bacteria consistent with Nocardia. The brain biopsy procedure was canceled. The patient was immediately placed on triple therapy antibiotics: IV sulfamethoxazole/ trimethoprim, imipenem/cilastatin and linezolid. Evaluation for underlying occult immunocompromised state including HIV, immunoglobulin levels, viral hepatitis panel, and uncontrolled diabetes mellitus were negative. Evaluation for other infectious etiologies, such as blood cultures, 1,3 beta-D-glucan, serum antigen testing for histoplasma, cryptococcus, and aspergillus were negative. The patient continued to stabilize over the course of his hospitalization and was eventually discharged to a rehabilitation facility to continue IV triple therapy with plans to consolidate antibiotics once final susceptibilities were reported and repeat imaging revealed improvement in CNS involvement.

\section{Discussion}

In the late 1800s, Edmond Nocard was first to isolate an aerobic filamentous organism from lesions in cattle suffering from "farcy" on Guadeloupe Island. ${ }^{5}$ One year later, the genus Nocardia was created to accommodate this new organism and the first species isolated was named Nocardia farcinica. ${ }^{6}$ It is now known that Nocardia is found ubiquitously in the soil worldwide, with modes of inoculation, including inhalation, ingestion, and transcutaneous spread. ${ }^{7}$ Since the initial discovery, over 50 different Nocardia species have been isolated. Of the various species, $N$. asteroides, $N$. farcinica, $N$. nova, and $N$. abscessus cause most of the invasive infections in humans. ${ }^{7.8}$

The first case of primary pulmonary nocardiosis occurring in a patient in the United States was described in 1898. ${ }^{5}$ A survey published in 1976, reported an incidence between 500-1,000 cases per year in the United States. ${ }^{10}$ Based on a review of over 1,000 cases, infection rates appear to have a male to female ratio of 2.4:1.0. ${ }^{5}$ This was likely an underestimate due to the challenges in accurate diagnosis, such as the relatively slow growth (sometimes up to 4 weeks) resulting in the cultures being discarded before the bacterial colony can be visualized. ${ }^{5}$ Although not part of the natural 


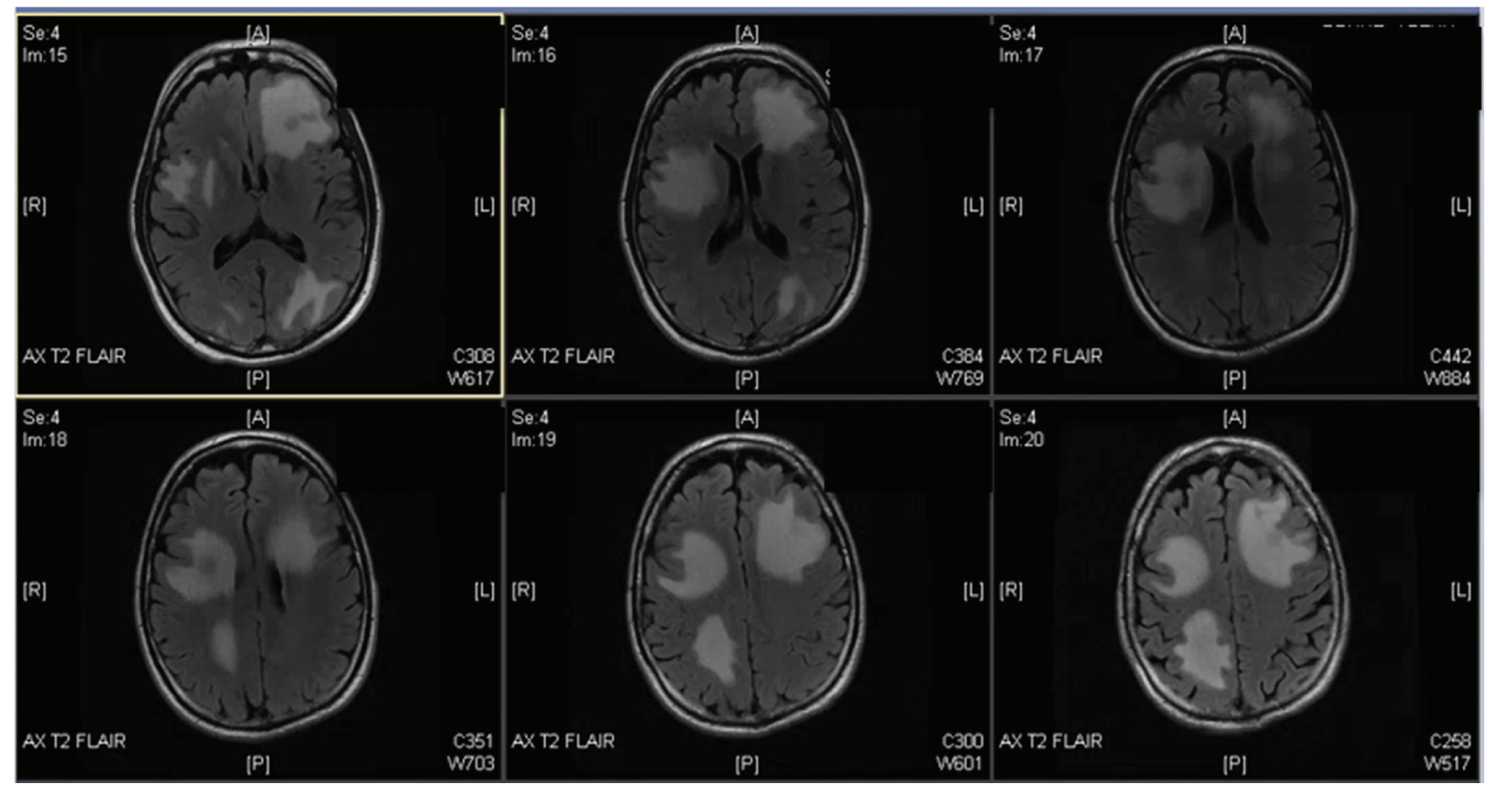

human microbiome, non-clinically significant culture positivity representing sub-clinical infections can be seen in up to $25 \%$ of healthy individuals. ${ }^{5}$ In this case, Nocardia was deemed pathogenic due to the fact that two separate tissue cultures grew the bacteria.

Although the incidence remains rare, more cases of clinically significant nocardiosis is on the rise, likely due to the higher prevalence of immunocompromised hosts, as well as improved modalities of identification in the laboratory. Solid organ transplant recipients are at the highest risk of developing nocardiosis with up to $3 \%$ of reported heart, kidney, liver and lung recipients suffering from infection. ${ }^{10}$ In a review of 5,126 solid organ transplant recipients, lung transplant recipients were found to have the highest incidence when compared to other organ recipients. ${ }^{11}$ Other risks factors include HIV, diabetes, cytomegalovirus, alcoholism, and structural lung disease, typically with chronic exposure to systemic corticosteroids., ${ }^{4,12}$ Despite being deemed as an opportunistic infection, one-third of reported nocardiosis cases are in patients who are immunocompetent. ${ }^{2}$ It is paramount to have a high suspicion for nocardiosis, even in an immunocompetent host, as this disease carries a mortality rate of up to $64 \%{ }^{4}$

There are six forms of disease caused by Nocardia in humans: pulmonary nocardiosis, systemic (disseminated) nocardiosis (involving two or more body sites), CNS nocardiosis, extrapulmonary nocardiosis, cutaneous and subcutaneous/lymphocutaneous nocardiosis and actinomycetoma. ${ }^{5}$ Most commonly, patients present with isolated pulmonary nocardiosis. ${ }^{12}$ Thirty-two percent of cases present as systemic infection, and of these, $44 \%$ have concomitant CNS involvement. ${ }^{5}$ Although there are various clinical manifestations, most cases present as pneumonia (acute, subacute or chronic) with associated findings, such as fever, weight loss, dyspnea, cough, and possible hemoptysis. Radiographic findings can vary from lobar infiltrates, effusion, abscesses, cavities, lobar consolidations, subpleural plaques, nodules, and endobronchial masses. ${ }^{3}$ Typically, there is a lack of significant mediastinal lymphadenopathy. ${ }^{2}$

Regarding treatment, the antimicrobial regimen recommended is based upon expert opinion, as a prospective randomized trial may never be conducted due to the rarity of the disease. Choice of antibiotics, route and duration are dictated not only by site of infection, but also susceptibility patterns. In a 10-year retrospective study published in 2010, 765 Nocardia isolates were subjected to antimicrobial susceptibility testing, reporting $61 \%$ resistance rates to sulfamethoxazole and $42 \%$ resistance rates to trimethoprim-sulfamethoxazole. ${ }^{8}$ A more recent study, published in 2012, reports a much lower rate of sulfonamide resistance (2.5\%) when sampling 552 isolates. ${ }^{12}$ Sulfonamides remain recommended as monotherapy for mild to moderate isolated pulmonary nocardiosis. ${ }^{1,12}$ If resistance is present or if the patient is sulfa-allergic, an alternative regimen includes the use of amikacin plus one of the following: imipenem, meropenem, or ceftriaxone. ${ }^{14}$ For more severe disease, including disseminated and CNS nocardiosis, triple therapy to include IV sulfamethoxazole/trimethoprim, imipenem-cilastatin and amikacin has been suggested. 12,14 A total of 6-12 months of therapy is recommended, including an induction phase (comprised of IV triple therapy) of 3-6 weeks duration, that is then transitioned to a combination (at least two-drug regimen) oral therapy, to include a sulfonamide, minocycline and/or amoxicillin-clavulanate. ${ }^{14}$ Close follow-up and serial imaging are paramount to demonstrate improvement with ongoing treatment prior to switching to oral therapy.

This rare case of disseminated nocardiosis in an immunocompetent host, presenting with non-resolving pneumonia, an endobronchial mass and CNS involvement, illustrates the importance of accurate diagnosis and appropriate therapy in a potentially fatal infectious disease. 
Endobronchial nocardiosis has been described in the literature, with associated risk factors including immunocompromise, exposure to chronic corticosteroids, and structural lung disease (bronchiectasis, chronic obstructive pulmonary disease, interstitial lung disease); none of which were appreciated in this case. ${ }^{5}$ In this patient, due to the overall duration of illness, despite paucity of neurologic symptoms, we believe he likely developed sub-clinical CNS involvement prior to bronchoscopy that finally manifested due to mass-effect. The importance of brain imaging after diagnosis is paramount to effectively treat, as the recommended treatment regimen, including choice of antimicrobial agent, as well as duration, is predicated on clinical severity and organ-involvement.
Nocardiosis has been coined "the great imitator" due to the vast heterogeneity in clinical presentation. This case of disseminated nocardiosis presenting as an endobronchial lesion with life-threatening CNS involvement in an immunocompetent host only reinforces this claim. This reminds us, as clinicians, to maintain a broad differential and to not focus on the most common causes of the clinical radiographic presentation. Also, this case demonstrates the importance of obtaining prompt and adequate brain imaging once the diagnosis of pulmonary nocardiosis is made, given the high incidence of CNS involvement reported in the literature. ${ }^{7}$ As incidence rises, improving awareness and therefore accurate diagnosis will be imperative in combating this disease that will hopefully lead to appropriate treatment and therefore improved outcomes in this growing patient population.
1. Sorrel T, Mitchell DH, Iredell JR, Chen SCA. Nocardia species. In: Mandell GL, Bennett JE, Dolin R, eds. Principles and Practice of Infectious Diseases. 7th ed. Philadelphia: Churchill Livingstone/ Elsevier. 2010;3199Y3207.

2. Kumar N, Ayinla N. Endobronchial pulmonary nocardiosis. Mt Sinai J Med. 2006;73:617-9.

3. Abdel-Rahman N, Izhakian S, Wasser WG, et al. Endobronchial enigma: a clinically rare presentation of Nocardia beijingensis in an immunocompetent patient [published correction appears in Case Rep Pulmonol. 2016;2016:1950463]. Case Rep Pulmonol. 2015;2015:970548.

4. Martínez Tomás R, Menéndez Villanueva R, Reyes Calzada S, et al, Pulmonary nocardiosis: risk factors and outcomes. Respirology. 2007;12:394-400

5. Beaman BL, Beaman L. Nocardia species: host-parasite relationships. Clin Microbiol Rev. 1992;7:213-64.

6. Lechevalier HA. Nocardioform actinomycetes. In: Williams ST, Sharpe ME, Holt JG (ed.), Bergey's manual of systematic bacteriology, vol. 4. Baltimore, USA: The Williams \& Wilkins $\mathrm{Co}$. 1989;2348-404.

7. Anagnostou T, Arvanitis M, Kourkoumpetis T, et al. Nocardiosis of the central nervous system: Experience from a general hospita and review of 84 cases from the literature. Medicine (Baltimore). 2014;93:19-32.

8. Uhde KB, Pathak S, McCullum I Jr., et al. Antimicrobial-resistant Nocardia isolates, United States, 1995-2004. Clin Infect Dis. 2010;51:1445-8.

9. Beaman B, Burnside J, Edwards B, Causey W. Nocardial infections in the United States, 1972-1974. J Infect Dis. 1976;134:286-289.

10. Ambrosioni J, Lew D, Garbino J. Nocardiosis: updated clinical review and experience at a tertiary center. Infection. 2010;38:89-97

11. Peleg AY, Husain S, Qureshi ZA, et al. Risk factors, clinical characteristics, and outcome of Nocardia infection in organ transplant recipients: a matched case-control study. Clin Infect Dis. 2007;44:1307-14

12. Wilson JW. Nocardiosis: updates and clinical overview. Mayo Clin Proc. 2012;87:403-7.

13. Brown-Elliott BA, Biehle J, Conville PS, et al. Sulfonamide resistance in isolates of Nocardia spp. from a US multicenter survey. J Clin Microbiol. 2012;50:670-2.

14. Gilbert DN, Eliopoulos GM, Chambers HF, et al. The Sanford Guide to Antimicrobial Therapy 2019. Sperryville, Virginia, USA: Antimicrobial Therapy, Inc.; 2019.

15. Lerner PI. Nocardiosis. Clin Infect Dis. 1996;22:891-903. 